\title{
CCD photometry and astrometry of visual double and multiple stars of the HIPPARCOS Catalogue
}

\section{CCD photometry and differential astrometry of 288 southern "Intermediate" systems *}

\author{
J. Cuypers ${ }^{1}$ and W. Seggewiss ${ }^{2}$ \\ ${ }^{1}$ Koninklijke Sterrenwacht van België, Ringlaan 3, B-1180 Brussel, Belgium \\ 2 Observatorium Hoher List der Universitätssternwarte Bonn, D-54550 Daun, Germany
}

Received December 17, 1998; accepted May 6, 1999

\begin{abstract}
We present photometric and astrometric data of about 280 visual double stars of the "intermediate" class, i.e. with angular separations mainly in the range $2^{\prime \prime}<\rho<12^{\prime \prime}$. The observations have been obtained in 1991-92 with a CCD camera attached to the $91 \mathrm{~cm}$ Dutch telescope at ESO La Silla, Chile. Differential magnitudes of the double star components as well as magnitudes and colour indices of the individual components have been determined in the Cousins $V$ and $I$ passbands with an internal error of about $0.005 \mathrm{mag}$ and an external accuracy of less than $0.03 \mathrm{mag}$. In addition, angular separations have been secured to an internal accuracy of $0.004^{\prime \prime}$ and position angles to about $0.05^{\circ 1}$.
\end{abstract}

Key words: stars: binaries: visual — astrometry

\section{Introduction}

Already in August 1990 a European Network of Laboratories "Visual Double Stars" was founded (Oblak et al. 1992a) for combining the efforts of scientist in six European countries with the goal to achieve accurate ground-based photometric and astrometric data of visual double and multiple stars as a complement to HIPPARCOS space observations (Oblak \& Lampens 1992).

In 1992 an ESO key programme was established to support photoelectric and CCD observations of visual double

Send offprint requests to: J. Cuypers

* Based on observations collected at the European Southern Observatory, La Silla, Chile.

1 Tables 1 to 4 are only available in electronic form at the CDS via ftp to cdsarc.u-strasbg.fr (130.79.128.5) or via http://cdsweb.u-strasbg.fr/Abstract.html stars in the southern hemisphere (Oblak et al. 1992b). Short summaries of the observations at ESO La Silla have been given by Lampens et al. (1997) and Oblak et al. (1997). Here we report on CCD photometry and astrometry performed in 1991-92 before the start of the key programme observations. This report complements a series of more detailed presentations and discussions of the ESO Key Programme results (Oblak et al. 1999, Paper I).

\section{Observations}

The observations were obtained in 1991, October 18-23 by JC and in 1992, February 18-24 by WS at the $91 \mathrm{~cm}$ Dutch light collector at the European Southern Observatory (ESO), La Silla, Chile. The scale of the f/13.75 telescope is approximately $16.4^{\prime \prime} \mathrm{mm}^{-1}$. In both observing runs we used the coated GEC CCD chip ESO \#7 with $576 \times 385$ pixels. The pixel size of $22 \times 22 \mu \mathrm{m}^{2}$ leads to a theoretical scale of $0.361^{\prime \prime}$ pixel $^{-1}$. The use of filters can alter this value.

The Network team agreed to observe in two wavelength bands: $V$ and $I$. We took from the filters available in the ESO filter set Bessel $V$ and Gunn $i$ and used neutral density filters when necessary.

Some problems with the focusing of the telescope and camera adapter resulted in a loss of efficiency and a much smaller number of observations per night than expected.

Normally all programme stars were observed at least three times in each filter. We increased the number of exposures up to 20 with increasing brightness ratio of the binary components.

During these runs we observed 288 visual double stars:

- 277 stars from the HIPPARCOS Input Catalogue (HIC, Turon et al. 1992);

- 11 astrometric standard stars, 4 without HIC number; 
- 6 double stars without HIC numbers, identified by their BD number, and

- HD 214509, one of the photometric standards, which turned out to be a close visual double star.

The programme stars can be classified as "intermediate" double stars with separations normally in the range $2^{\prime \prime}<\rho<12^{\prime \prime}$. Four stars have separations $\rho$ less than $2^{\prime \prime}$ and 39 stars have separations greater than $12^{\prime \prime}$. The programme was limited to magnitude differences between the two components mainly in the range 0 mag $<\Delta V<3$ mag.

The final calibration process was performed on the basis of the HIPPARCOS data (see Sect. 3). As additional tool for defining scale and position angle astrometric standard stars were observed regularly. They have been taken from the lists of Brosche \& Sinachopoulos (1988 and 1989). In addition, trails of bright stars over the CCD frame were generated by stopping the telescope tracking.

$V$ and $I$ standard stars of the Cousins system with no indications of variability in the Geneva photometry were selected from the lists of Landolt (1983), Menzies et al. (1989) and Menzies et al. (1991) by Grenon (1991, private communication) and measured in each night of good photometric quality.

\section{Reduction and calibration}

All frames with double stars have been reduced with a profile-fitting reduction method developed by JC. A special method was necessary since the majority of our frames showed only the overlapping profiles of the double stars, but no further (single) stars on the frames to define the point spread function (for details: see Cuypers 1997). The astrometric standard stars and a few preliminary HIPPARCOS results were used for a first scale calibration $\left(0.378^{\prime \prime} \mathrm{pixel}^{-1}\right)$. The final calibration of separations and position angles was performed on the basis of all available corresponding HIPPARCOS data (ESA, 1997) by minimizing the sum of the squares of all the differences in relative positions. Stars with large discrepancies in position ( $3 \sigma$-level) between the CCD and HIPPARCOS measurements were not included in the final calculation of the transformation, but they can be found in the tables of results.

The final scale correction was 0.9969 for 1991 and 0.9968 for 1992 and the position angle correction $1.280^{\circ}$ in 1991 and $3.063^{\circ}$ in 1992 .

Most nights were of sufficient photometric quality. All instrumental magnitudes taken during these nights through the Bessel $V$ and Gunn $i$ filters were, after correction for atmospheric extinction, transformed into the Cousins $V$ and $I$ standard system with the aid of the observed standard stars. The differences between both pairs of standard bands $(V, i)$ vs. $(V, I)$ are not negligible but they could be treated safely by the transformation equations.

\section{Results}

The results are listed in Tables 1 to 4 (available electronically via the CDS) in sequence of the HIC numbers of the programme stars. Astrometric standard stars with a HIC number are filed among the programme stars, but can easily be identified by their large number of observations. If they have no HIC number then they are identified by their BD numbers and listed together with 6 additional double stars at the end of the tables. In addition, one photometric standard star, HD 214509, turned out to be a close visual double star (last star in all tables) with separation $\rho=1.241^{\prime \prime}$ and magnitude difference $\Delta V=2.649 \mathrm{mag}$. Tables 1 and 3 give summarizing results, whereas the individual observations of the stars that were observed in more than one night are listed in Tables 2 and 4.

Table 1 lists the differential astrometric and photometric results. One can find an identifier, the date of the observation, the total number of observations $(N)$, the mean separation $\rho$ and the mean position angle $\theta$, the number of observations in $V\left(N_{V}, N_{i}\right)$ and the (instrumental) magnitude differences $\Delta V$ and $\Delta i$ between the components $\mathrm{A}$ and $\mathrm{B}$ of the double stars. The values are quoted with their standard error of the mean $(\sigma)$. The "distance" between the binary parameters in $V$ and $i$ (in arcsec) is given in the column $r_{V, i}$ :

$r_{V, i}=\sqrt{\rho_{V}^{2}+\rho_{i}^{2}-2 \rho_{V} \rho_{i} \cos \left(\theta_{V}-\theta_{i}\right)}$.

This value is important for the judgement of the quality of the measurements. It will be discussed in the next section.

The distances $r_{\mathrm{N}, \mathrm{H}}$ between the listed observations of the Network $(\mathrm{N})$ and the HIPPARCOS results $(\mathrm{H})$, defined as

$r_{\mathrm{N}, \mathrm{H}}=\sqrt{\rho_{\mathrm{N}}^{2}+\rho_{\mathrm{H}}^{2}-2 \rho_{\mathrm{N}} \rho_{\mathrm{H}} \cos \left(\theta_{\mathrm{N}}-\theta_{\mathrm{H}}\right)}$

are given as well. In general an extremely good agreement is found. A detailed discussion of the comparison with the Hipparcos data will be postponed to a subsequent paper.

Table 3 gives more details of the final photometric results: we list the standard $V$ magnitudes and the colour indices $V-I$ of the components A and B together with the standard errors of the mean on these quantities. Also given are the number of observations in each filter $\left(N_{V}, N_{i}\right)$ and the differences $\Delta(V-I)$ between the colours of the components.

Two systems have secondary components brighter than the primaries in comparison to the designation in earlier catalogues of binaries, i.e. $\Delta V<0.0 \mathrm{mag}$, namely HIC 44711 (-0.015 mag) and HIC 80245 (-0.017 mag), but this is, of course, filter dependent. In some cases a different faint secondary component was measured instead of the component indicated in earlier catalogues: for example: HIC 39056 with $\Delta V=5.939$ mag.

The magnitude range of the primary components in the sample is mainly $7.5 \mathrm{mag}<V_{\mathrm{A}}<10.0 \mathrm{mag}$; one star is brighter and 21 stars are fainter than these limits. 


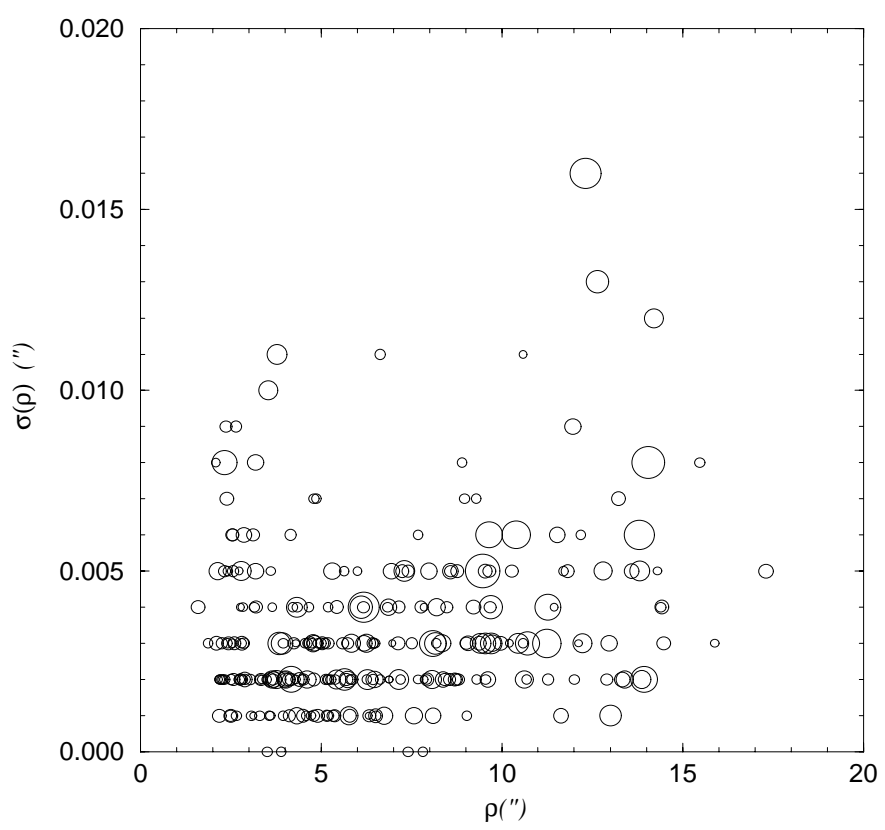

Fig. 1. Internal errors $\sigma(\rho)$ of the angular separation vs. mean separation $\rho$ and differential magnitude $\Delta V$ of the binary components (larger symbols indicate larger $\Delta V$ )

Twenty three pairs have instrumental $\Delta V$ values in excess of $3.0 \mathrm{mag}$ reaching a maximum of $\Delta V=5.939 \mathrm{mag}$.

The colour indices $V-I$ of the A components span the range from -0.164 to $+3.274 \mathrm{mag}$, i.e. from early B-type to late M-type stars (neglecting possibly present reddening and a few "outliers"). The same holds for the B components where $V-I$ runs from -0.178 to $+3.291 \mathrm{mag}$. The differences $\Delta(V-I)$ of the colours $V-I$ of the binary components are negative in 45 systems. Astrophysical implications of this will be discussed in other papers.

\section{Discussion of errors}

\subsection{Internal astrometric consistency}

The large number of repeated observations of the individual stellar systems (Table 2) allows to estimate very well the internal astrometric and photometric consistencies.

The overall mean standard deviation (s.d.) in separation amounts to $\sigma(\rho)=0.004^{\prime \prime}$. This corresponds to about $0.01 \times$ the pixel width of the CCD. The small value is, of course, the consequence of the repeated measurements for the program stars and was confirmed by the preliminary results of the other observational runs of the network (Lampens et al. 1997). The mean s.d. in position angle $\sigma(\theta)=0.05^{\circ}$.

The dependence of the s.d. in separation $\sigma(\rho)$ from the separation $\rho$ and from the magnitude difference $\Delta V$ is illustrated in Fig. 1. The s.d. in separation between $4^{\prime \prime}$ and $12^{\prime \prime}$ is apparently not much dependent on the separation itself. Only for the systems with $\rho<4^{\prime \prime}$ and $\rho>12^{\prime \prime}$

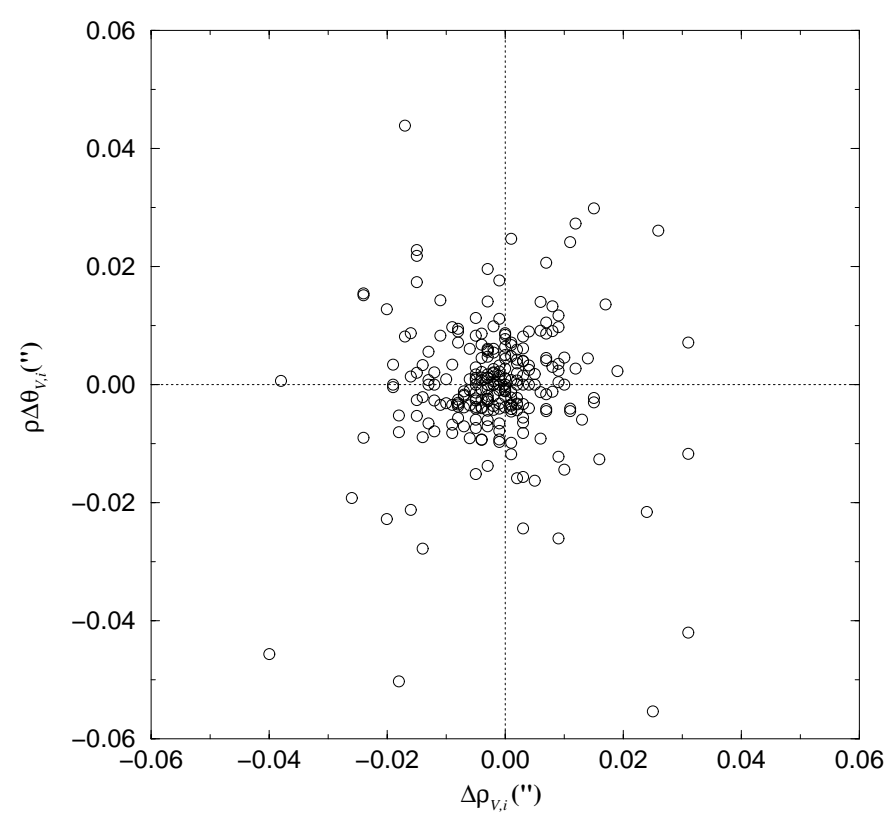

Fig. 2. Differences between the astrometry in $V$ and $i\left(\rho\left(\theta_{V}-\right.\right.$ $\left.\theta_{i}\right)=\rho \Delta \theta_{V, i}$ versus $\left.\Delta \rho_{V, i}\right)$

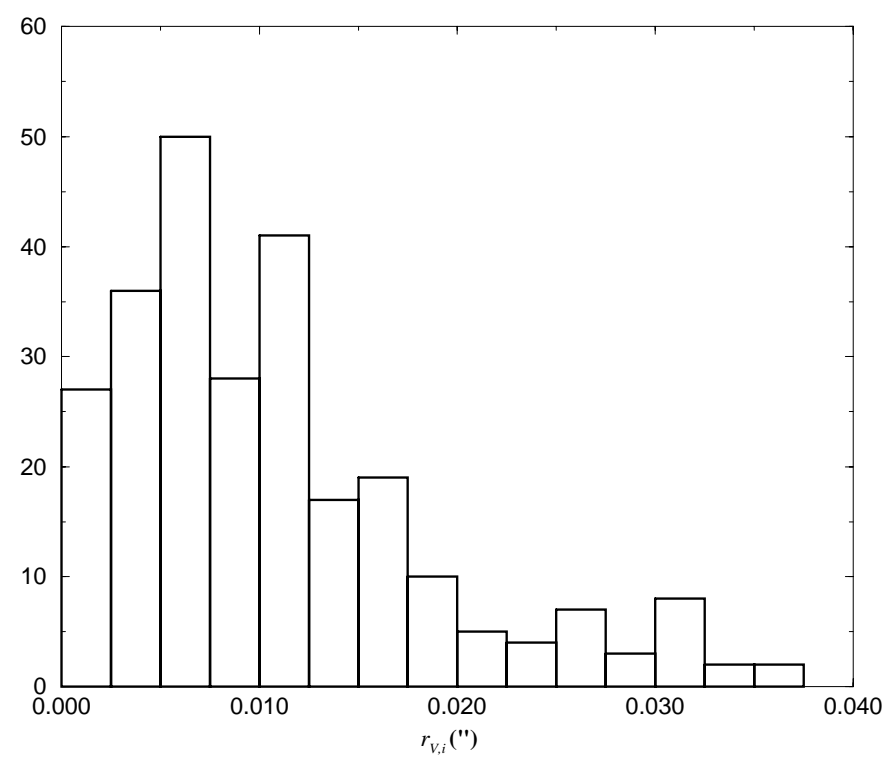

Fig. 3. Histogram of the mean positional differences $r_{V, i}$

an increase of the s.d. in separation is noticed. The small separations are close to the seeing limit and at larger separation the isoplanicity could be lost, resulting in a small degradation of the results for $\rho>12^{\prime \prime}$.

The difference between astrometric parameters of our CCD observations in the passbands $V$ and $i$ is a check for the internal consistency. Taking the mean over all binary systems, the separation in the $V$ band is $0.002^{\prime \prime}$ smaller than in $i$. The standard deviation of the differences in separation is $0.010^{\prime \prime}$. The mean difference in position angle between $V$ and $i$ is $0.01^{\circ}$ or $0.0012^{\prime \prime}$ if expressed as $\rho \Delta \theta$ 


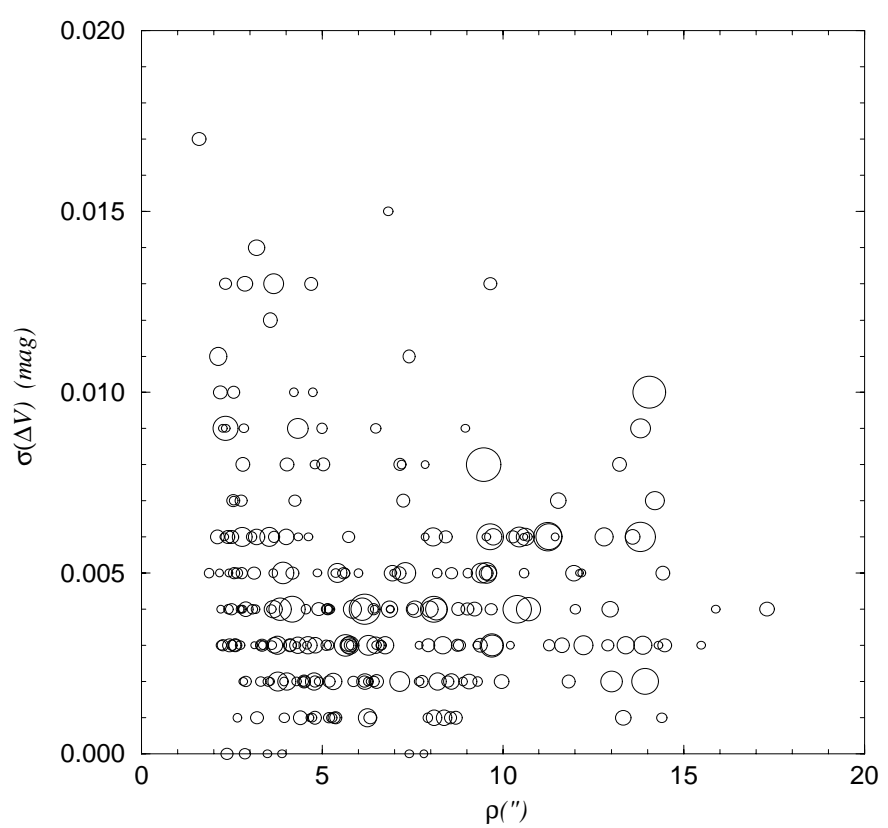

Fig. 4. Internal errors $\sigma(\Delta V)$ of the differential magnitude vs. mean separation $\rho$ and differential magnitude $\Delta V$ (larger symbols indicate larger $\Delta V$ )

(s.d. is $\left.0.011^{\prime \prime}\right)$. No indications of systematic differences were found, as can be seen in Fig. 2.

The quality of the measurements can also be read off the distances $r_{V, i}$ as defined in Eq. (1). The value $r_{V, i}$ is only zero if both, $\rho_{V}=\rho_{i}$ and $\theta_{V}=\theta_{i}$. The overall mean distance $\overline{r_{V, i}}$ is $0.011^{\prime \prime}$. This is in agreement with the value expected according to the error propagation over $r_{V, i}$. The distribution of these distances is shown in Fig. 3.

Measurements of the same system on more than one night or in different seasons provide also a very good internal consistency check. The mean of the deviations from the mean value of the separation for each system tabulated in Table 2 is only $0.004^{\prime \prime}$. This indicates again the very high level of accuracy attained in the differential astrometry.

Systems with high internal errors in separation and position angle (not included in the statistics) have almost always separations close to the seeing limit (e.g. HIC 18414 and the photometric standard HD 214509).

\subsection{Internal photometric consistency}

The internal photometric errors (of the magnitude differences in $V$ and $i$ ) are listed in Table 1. Their overall mean values are small: $0.005 \mathrm{mag}$ in $\Delta V$ and $0.006 \mathrm{mag}$ in $\Delta i$. Again, the large number of individual observations (especially increasing for stars with increasing $\Delta V$ ) is the reason of the small errors. Figure 4 displays the internal errors as a function of angular separation $\rho$ and magnitude difference $\Delta V$. No real tendency in one or the other direction can be seen, except that there are some larger errors (exceeding $0.01 \mathrm{mag}$ ) at small separations.

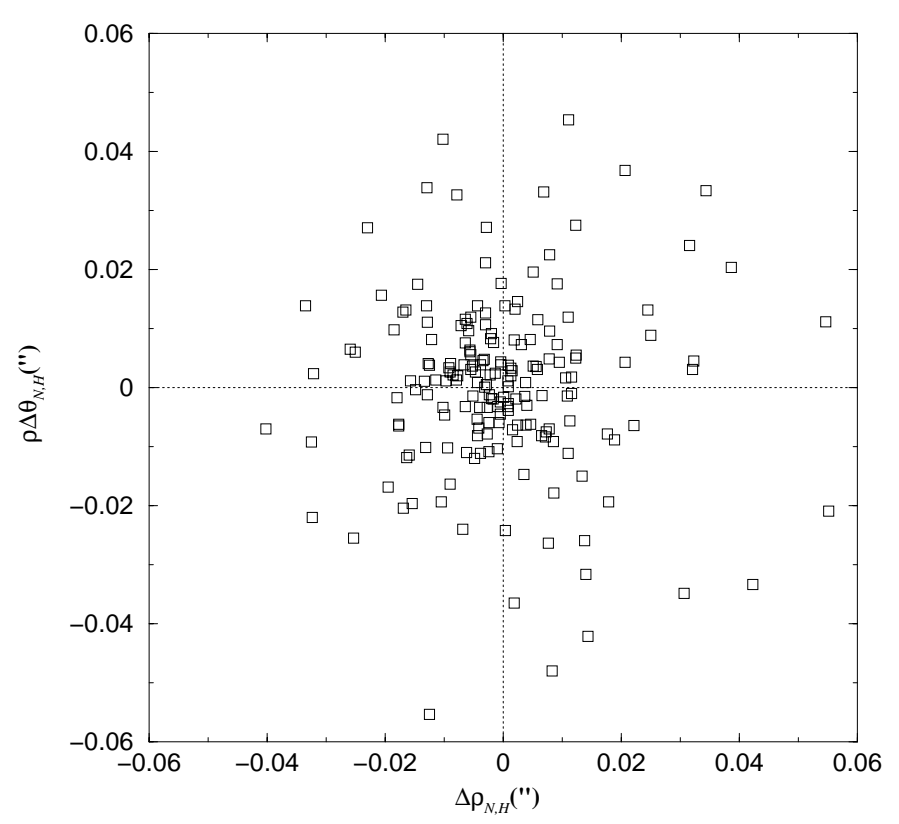

Fig. 5. Differences between the Hipparcos and the Network astrometry $\left(\rho\left(\theta_{\mathrm{N}}-\theta_{\mathrm{H}}\right)=\rho \Delta \theta_{\mathrm{N}, \mathrm{H}}\right.$ versus $\left.\Delta \rho_{\mathrm{N}, \mathrm{H}}\right)$

An independent check on the magnitudes can be done with the data of systems measured more then once (Tables 2 and 4 ). The mean value of the deviations from the mean of the differential values in $V$ is $0.009 \mathrm{mag}$ (median: $0.006 \mathrm{mag}$ ) and $0.016 \mathrm{mag}$ in $i$ (median: $0.006 \mathrm{mag})$. The standard photometry of the components is very compatible: mean values of the deviations on components $\mathrm{A}$ or $\mathrm{B}$, in $V, I$ or $V-I$ are in the range 0.014 to $0.021 \mathrm{mag}$.

The cited mean values could even be a too high estimate of the errors, since variability for some of the components is not excluded and some systems were measured again only because there was some doubt on the quality of the first observations.

\subsection{Comparison with astrometric catalogue data}

The discussion of the final comparison of the observations with the HIPPARCOS data will be presented elsewhere (Oblak et al., in preparation). Here we again point out that the calibration of our data was performed with the separations and position angles measured by HIPPARCOS (see Sect. 3). Therefore, the mean of the differences between the HIPPARCOS and the Network astrometry in the $(\rho, \theta)$ plane (see Fig. 5$)$ is zero. The standard deviations of these differences are $0.014^{\prime \prime}$ in separation and $0.18^{\circ}$ in position angle (or $0.016^{\prime \prime}$ if expressed as $\rho \Delta \theta$ ), respectively. For the distances $r_{\mathrm{N}, \mathrm{H}}$ between the HIPPARCOS and the Network results, as defined in Eq. (2), we get a mean value of 0.019 ", if neglecting the "outliers". The forgoing errors and distances display again the high quality of the Network results. 


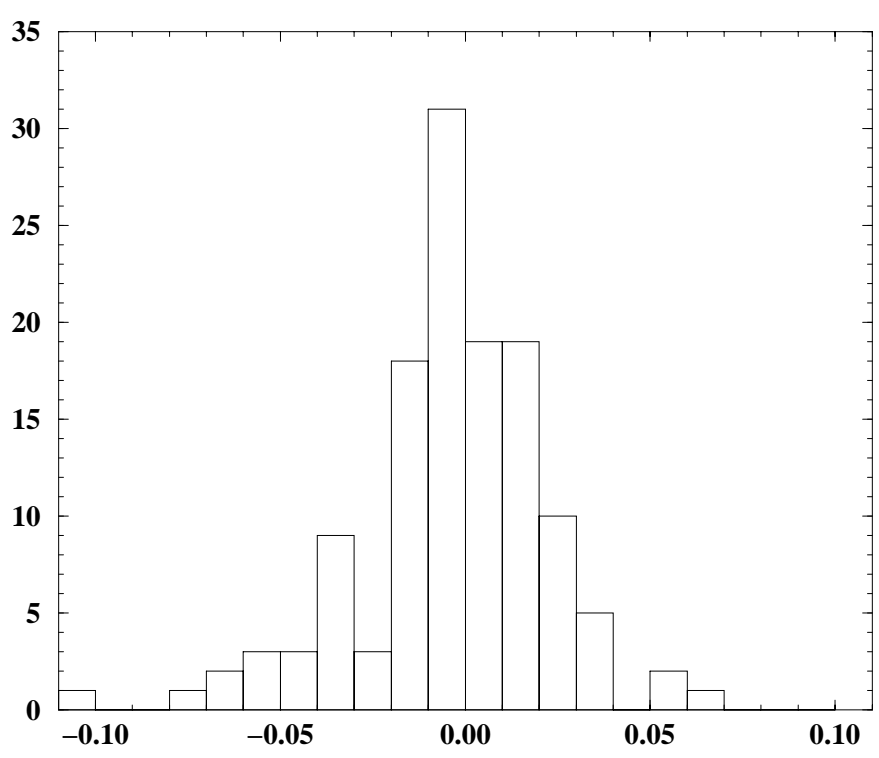

Differences of joint magnitudes (CCD- photoelectric V)

Fig. 6. Histogram of the differences in joint magnitudes between CCD photometry and photoelectric photometry $(V)$

In addition we report briefly on the comparison with the HIC annex (Turon et al. 1992, Vol. 6), which contains data mainly from the "Catalogue des Composantes d'Etoiles Doubles et Multiples" (CCDM, Dommanget \& Nijs 1995). The following statements throw, of course, more light onto the reliability of the data before the "CCD era" than on the recent observations. In any case one must admit that the astrometric and photometric characteristics may change due to binary motion, proper motion and light variability.

The mean difference between the angular separations CCD minus HIC is $0.1^{\prime \prime}$ (leaving out here and in the following text a few very discordant values, probably caused by misidentifications). The median value is only $0.03^{\prime \prime}$, but the standard deviation on the differences is $0.8^{\prime \prime}$. No systematic effects could be be noticed.

The scatter of the HIC separations around the CCD values is increasing for separations in excess of $8^{\prime \prime}$. Likewise the scatter increases for small $(\Delta V<1.0 \mathrm{mag})$ and large $(\Delta V>3.0 \mathrm{mag})$ magnitude differences between the double star components.

For several stellar systems the position angle differs by about $180^{\circ}$. This simply means that the new measurements and the reduction interchanged the components $\mathrm{A}$ and $\mathrm{B}$ : the former component $\mathrm{B}$ is now the brighter one. In the mean, the HIC position angles are the same as the CCD ones (mean difference: $0.1^{\circ}$, median $0.0^{\circ}$ ), but the scatter is very large: about $6^{\circ}$.

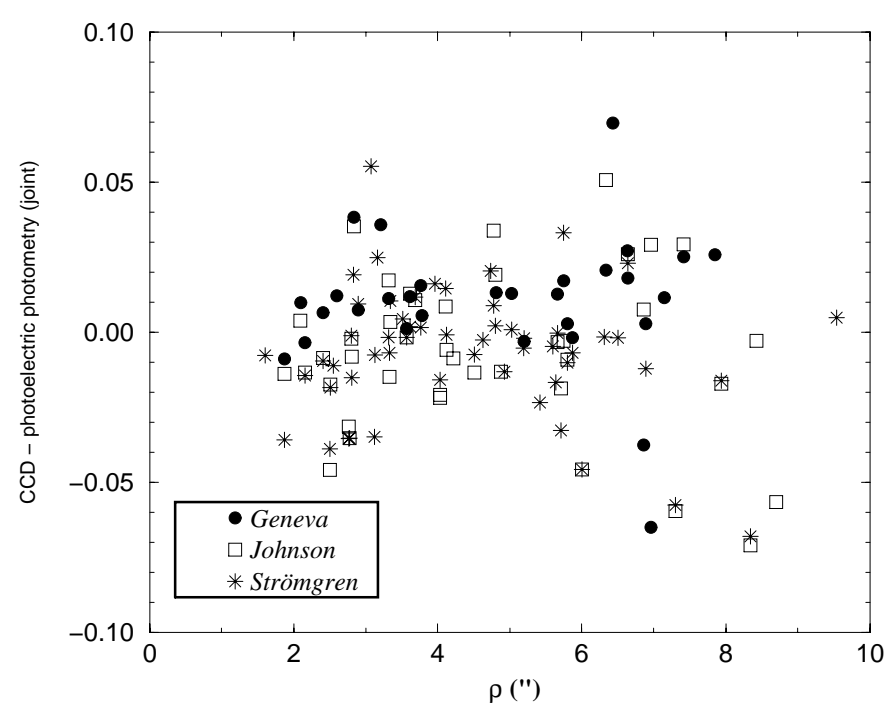

Fig. 7. Differences in joint magnitudes between CCD photometry and photoelectric photometry vs. mean separation $\rho$

\subsection{Comparison with published magnitude data and photoelectric measurements}

As sources for the magnitudes of double stars and double star components we used the annex of the HIC (Vol. 6), where component magnitudes are given to a tenth of a magnitude as in the CCDM, and the HIC itself. In the HIC mostly joint photometry is given, but here we can select the most reliable measurements by means of the error bars.

No systematic differences were found between our CCD measurements and the estimates of the magnitudes of the main components A as listed in the HIC annex. The mean value of the differences is $-0.05 \mathrm{mag}$ (median is $-0.03 \mathrm{mag})$, but the scatter is large $(0.4 \mathrm{mag})$ and values range from -1.7 to $1.1 \mathrm{mag}$. The estimates of the magnitude differences between components $\mathrm{A}$ and $\mathrm{B}(\Delta V)$ differ largely from the CCD measurements: from -1.0 to $+1.1 \mathrm{mag}$ with a s.d. of $0.3 \mathrm{mag}$, if we do not take probable misidentifications into account. The mean of $0.1 \mathrm{mag}$ could be significant but we have no explanation for this.

For a few binary stars of our sample individual photometric data on the A components are now available in the literature, e.g. in the Lausanne General Catalogue of Photometric Data (Mermilliod et al. 1997) or in the Besançon Data Base of Double and Multiple Stars (Kundera \& Oblak 1998). Some were already given in the HIC, but it is not always clear whether an entry is joint photometry or the A component magnitude. The indication of the sources of the $V$ magnitude in the HIC is not complete: amongst the codes we find $\mathrm{D}$ for joint and $\mathrm{P}$ for photoelectric. As a consequence we could not always decide whether a photoelectric measurement was joint or only the A component.

We found 14 stars with errors smaller than 0.05 mag where the published $V$ magnitudes refer without doubt 


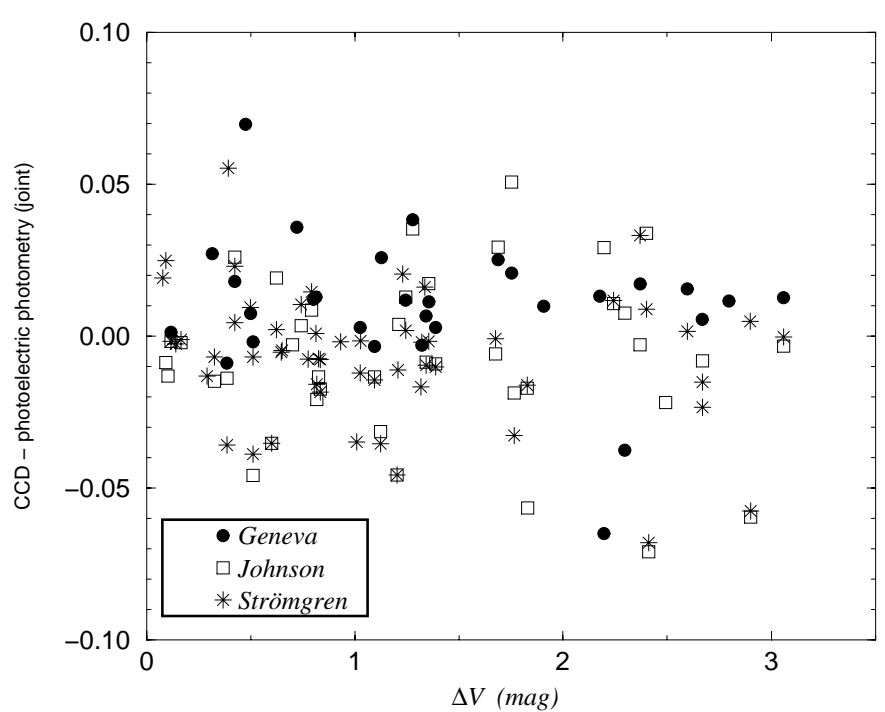

Fig. 8. Differences in joint magnitudes between CCD photometry and photoelectric photometry vs. difference $\Delta V$ in magnitude between components

to the A components. The source of the magnitudes is mostly photoelectric. The mean of the deviations with our CCD data is only 0.002 mag with a 0.04 mag scatter. Since the mean error on the published magnitudes is about $0.03 \mathrm{mag}$, this is in agreement with a similar error on our component photometry.

More comparison data are available for the joint photometry, i.e. for magnitudes taken of both components in common with a sufficiently large diaphragm. We found 30 double stars with Geneva $(V), 42$ with Johnson $(V)$ and 55 with Strömgren $(y)$ joint photometry in our sample. Several double stars have measurements in the three photometric systems with differences of about 0.01 mag.

No systematic difference between the CCD and photoelectric observations could be detected. The mean difference (in the sense CCD minus photoelectric measurements) is $0.010 \mathrm{mag}$ for the Geneva data, $-0.009 \mathrm{mag}$ for the Johnson data and -0.007 mag for the Strömgren data. All are close to zero. The standard deviations of the differences are very small: 0.022, 0.030 and 0.022 mag respectively. A histogram with all data combined is given in Fig. 6. If we consider the errors on the published Geneva and Strömgren photometry as negligible and, as a consequence, estimate the error on the Johnson photometry as $0.02 \mathrm{mag}$, a good estimate for our CCD joint photometry error is $0.022 \mathrm{mag}$. This proves the high quality of our photometric data.

Although there is no overall dependence on separation, a few cases with angular separations above $6^{\prime \prime}$ and large magnitude differences between the components, have fainter photoelectric magnitudes (Figs. 7 and 8). This could be caused by loss of light when the two components were measured photoelectrically in a (too) small diaphragm.

\section{Conclusions}

We have obtained accurate and homogeneous CCD photometric data of 276 visual double stars of the HIPPARCOS Input Catalogue. The data include Cousins $V$ and $I$ magnitudes and colour indices $V-I$ for the individual double star components as well as the differences between the components. The internal errors of these differences are only $0.005 \mathrm{mag}$ in $V$ band and $0.006 \mathrm{mag}$ in the $I$ band. After an elaborated error analysis, including comparison with joint magnitudes of binary systems measured in different photometric systems (Johnson $U B V$, Geneva, Strömgren) we arrive at an external error not larger than 0.030 mag.

Angular separations $\rho$ and position angles $\theta$ have been secured for 288 double stars. As a consequence of the repeated measurements (up to 15 or more for increasing brightness ratios of the components), the internal errors are very small: $0.004^{\prime \prime}$ in separations, $0.05^{\circ}$ in position angles and $0.011^{\prime \prime}$ in position differences between measurements in different filters. A preliminary look at the positional differences between the CCD and Hipparcos data indicates very good agreement and the errors on the differential CCD astrometry seem to be as small as the Hipparcos data. This will be quantified in subsequent papers.

Acknowledgements. We thank Patricia Lampens (Brussels) and Edouard Oblak (Besançon) for their assistance in the "Visual Double Stars" programme and for the many enlightening discussions. JC acknowledges funding by project 2.0060 .91 of the Fund for Scientific Research (F.W.O.) - Flanders (Belgium).

\section{References}

Brosche P., Sinachopoulos D., 1988, Bull. Inf. CDS 34, 39 Brosche P., Sinachopoulos D., 1989, Bull. Inf. CDS 36, 3

Cuypers J., 1997, in Visual Double Stars: Formation, Dynamics and Evolutionary Tracks, Docobo J.A., Elipe A., McAlister H. (eds.), ASSL 223, 35

Dommanget J., Nys. O., 1995, Bull. d'Inf. du CDS de Strasbourg 46, 3

ESA, 1997, SP-1200, "The Hipparcos and Tycho Catalogues"

Kundera T., Oblak E., 1998, A General Data Base of Double and Multiple Stars,

http://bdb.obs-besancon.fr/bdb/Welcome.html

Lampens P., Cuypers J., Oblak E., Seggewiss W., Duval D., 1997, in Visual Double Stars: Formation, Dynamics and Evolutionary Tracks, Docobo J.A., Elipe A., McAlister H. (eds.), ASSL 223, 439

Landolt A.U., 1983, AJ 88, 439

Menzies J.W., Cousins A.W.J., Banfield R.M., Laing J.D., 1989, SAAO Circ. 13, 1

Menzies J.W., Marang F., Laing J.D., 1991, MNRAS 248, 642

Mermilliod J.C., Hauck B., Mermilliod M., 1997, A\&AS 124, 349 
Oblak E., Argue A.N., Cuypers J., Lampens P., Seggewiss W., et al., 1992a, in Complementary Approaches to Double and Multiple Star Research, IAU Coll. 135, McAlister H.A. and Hartkopf W.I. (eds.), ASP Conf. Ser. 32, 454

Oblak E., Argue A.N., Cuypers J., Lampens P., Seggewiss W. et al., 1992b, ESO Messenger 69, 14

Oblak E., Lampens P., 1992, in Complementary Approaches to Double and Multiple Star Research, IAU Coll. 135,
McAlister H.A. and Hartkopf W.I. (eds.), ASP Conf. Ser. 32,339

Oblak E., Lampens P., Cuypers J., et al., 1997, in Proceedings of the Hipparcos Venice '97 Symposium, ESA SP-402, 445

Oblak E., Lampens P., Cuypers J., et al., 1999, A\&A 346, 523 (Paper I)

Turon, et al., 1992, ESA-SP 1136, The Hipparcos Input Catalogue 